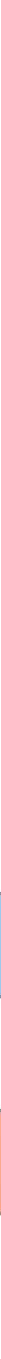




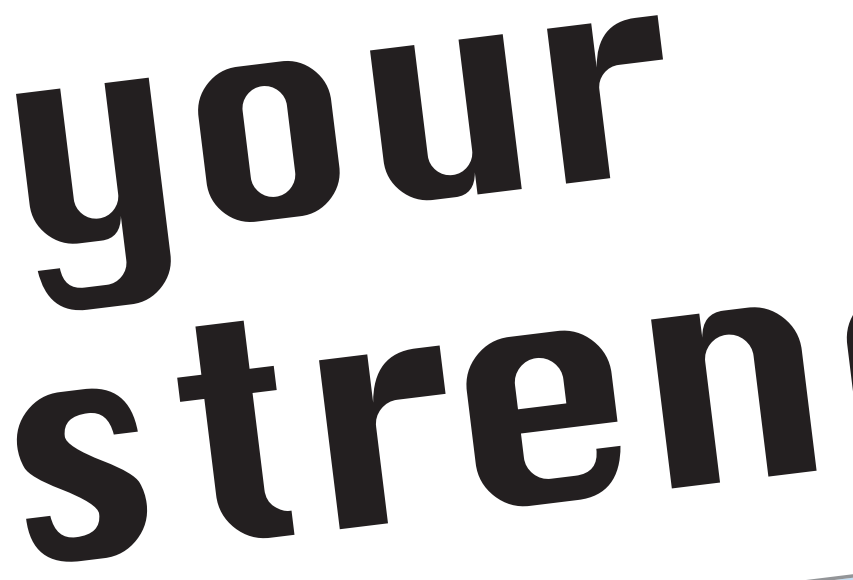

\section{get active}

forearms and balls of feet are now in contact with the floor.
The aim is to maintain a flat line with a small arch in your lower back, not tummy dropped down or backside in the air. Maintain the position for as long as is comfortable and aim to increase the time you can do this before the body begins to shake.

\section{Cat stretch}

Assume the all fours position, hands directly under shoulders, knees directly under hips. Without moving the line of the arms or legs, slowly contract the abdominal muscles aiming to hunch the back, lower spine shifting upwards. Hold for a second at maximum height then slowly release and lower the spine to exaggerate the lumber curve, abs down to the floor.

\section{Roll with it}

Lie on an exercise mat (or towel) on your back with your head in a neutral position so as not to unduly stress the cervical vertebrae. Lift one knee and then the other to your chest, heels down by the bum. Keeping your lower back in contact with the floor, wrap the arms around the legs and pull the thighs in towards the chest and hold for 15 seconds. If comfortable, gently roll backwards and forwards.

\section{Let's twist again}

Again, flat on your back, knees to chest but this time arms out to the side. Slowly lower both knees to the left side and simultaneously take the left arm to meet the right and look right. Hold for 15 seconds, return to middle position, then repeat on the opposite side.

\section{General Tips}

- Ensure active retaxation forms part of your exercise regime as flopping in front

of the television does not count as a stress buster. Reduce tension by using breathing techniques, such as those found in yoga and tai chi. Maybe even pay (or persuade!) someone to give you a good massage.

- Develop postural awareness by taking a class in either Alexander, Pilates or Feldenkrais techniques which can be found at health clubs or dance studios.

- Always bend your knees, not your back, when lifting and keep your abdominals pulled in tight as you exert.

- Carry a rucksack rather than a hand held bag, to evenly distribute the load.

- If you are a health club member ensure you are exercising safely by asking an instructor to observe your technique in the gym.

- Since working on a flat surface, such as a desk, has been highlighted as possibly predisposing problems, a writing slope is a good idea. It's a simple Perspex/wooden device allowing you to work at a more comfortable angle. Of course, every decent work station should also have a seat with adjustable height and back.

\section{!)}

